Revue d'histoire de l'Amérique française

REVUE D.HISTOIRE DE L'AMÉRIQUE FRANÇAISE

\title{
Camille Roy et Lionel Groulx : la querelle de L’Appel de la race
}

\section{Lucie Robert}

Volume 32, numéro 3, décembre 1978

Lionel Groulx, $100^{\mathrm{e}}$ anniversaire de sa naissance, 1878-1978

URI : https://id.erudit.org/iderudit/303717ar

DOI : https://doi.org/10.7202/303717ar

Aller au sommaire du numéro

Éditeur(s)

Institut d'histoire de l'Amérique française

ISSN

0035-2357 (imprimé)

1492-1383 (numérique)

Découvrir la revue

Citer cet article

Robert, L. (1978). Camille Roy et Lionel Groulx : la querelle de L'Appel de la race. Revue d'histoire de l'Amérique française, 32(3), 399-405.

https://doi.org/10.7202/303717ar d'utilisation que vous pouvez consulter en ligne.

https://apropos.erudit.org/fr/usagers/politique-dutilisation/ 


\title{
CAMILLE ROY ET LIONEL GROULX : la querelle de L'Appel de la race
}

\author{
LUCIE ROBERT \\ Université Laval \\ Québec
}

Sous le pseudonyme d'Alonié de Lestres - du nom d'un des compagnons de Dollard des Ormeaux - Lionel Groulx publiait en septembre 1922 un roman qui fut aussitôt un succès de librairie. En effet, la première édition de L'Appel de la race, tirée à 3300 exemplaires, fut épuisée en un mois. En moins de cinq mois, 6000 exemplaires étaient vendus. L'Appel de la race aura une troisième édition, puis une quatrième et trois autres dans la collection du "Nénuphar» de la maison Fides. En 1965, plus de quarante ans après sa parution, il fête son $20 \mathrm{e}$ mille. L'Appel de la race fut de plus publié en feuilleton et en bandes dessinées dans plusieurs journaux comme Le Bien public, Le Courrier de Saint-Hyacinthe, L'Action catholique, L'Évangéline et bien d'autres. Succès comme on en connut peu dans l'histoire de l'édition au Québec.

Mais cela ne se fit pas sans mal. L'Appel de la race, que son auteur avait publié sous un pseudonyme "pour ne pas compromettre [sa] gravité d'historien "1 suscita immédiatement les plus vives passions. La "bataille" dura huit mois et provoquera encore quelques propos cinglants en 1939, soit dix-sept ans plus tard.

L'intrigue est la suivante. Avocat en vue à Ottawa, Jules de Lantagnac ressent, à l'âge de 40 ans, l'appel de la race qu'il a presqu'abandonnée depuis sa jeunesse à l'Université McGill et son mariage à Maud Fletcher, une anglaise convertie au catholicisme. Conseillé par le père Fabien, un oblat de Hull, il retourne au lieu de son enfance, la campagne de Vaudreuil, tente de refranciser ses enfants et se lance à corps perdu dans la lutte des écoles catholiques de l'Ontario en se faisant élire député indépendant du comté de Russell au parlement fédéral. Vient le jour de la grande offensive qui

1 Lionel Groulx, Mes Mémoires (4 vol., Fides, Montréal 1970-1974), II : 87. 
doit porter le problème du règlement XVII dans l'arène fédérale. Devant l'attitude de Maud, qui ressent elle aussi l'appel de sa race, de Lantagnac hésite à prendre la parole au cours du débat. Sa femme menace de le quitter s'il s'implique. Mais le père Fabien balaie ses doutes en utilisant l'argument du "volontaire indirect". Jules de Lantagnac fait son discours; Maud Fletcher part avec deux de ses enfants. La seule consolation de Jules de Lantagnac après le départ de sa seconde fille pour le noviciat est la fidélité de son fils aîné à la race française de ses aïeux.

Se prononcent en faveur de la thèse du roman de Lionel Groulx: les milieux francophones de l'Ontario et de l'ouest du Canada à qui L'Appel de la race rappelle les dures batailles scolaires et le danger constant d'assimilation; l'A.C.J.C. (Association canadienne de la jeunesse catholique); la revue, L'Action française, qui soutient son directeur; Léo-Paul Desrosiers, futur romancier; Antonio Perrault, avocat, l'un des directeurs de l'Action française, et surtout Olivar Asselin qui intervient par une retentissante conférence prononcée à Montréal, le 15 février 1923. Avec eux, Jean-Marie-Rodrigue, futur cardinal Villeneuve, qui, contre toutes les attaques, soutiendra la théologie du père Fabien. Tous - et bien d'autres - font ressortir le caractère patriotique de l'œuvre.

Se prononcent contre L'Appel de la race, entre autres, Louvigny de Montigny, journaliste, fonctionnaire et écrivain et René du Roure, professeur de français à McGill, qui accusent l'auteur d'avoir écrit un roman à clé violant la vie privé d'un homme politique encore vivant à cette époque, le sénateur Belcourt. Contre aussi, Claude-Henri Grignon, futur auteur d'Un homme et son péché qui, sous le pseudonyme de Valdombre, qualifie l'ouvrage de roman manqué, «à cause du style d'abord, puis à cause du fond, de la collante thèse, sans conclusion et sans morale, histoire invraisemblable dont [il] ne distingue pas très bien l'utilité $»^{2}$. L'abbé Arthur Maheux, professeur au Séminaire de Québec et à l'Université Laval, conteste les affirmations de Groulx quant à l'absence de patriotisme dans l'enseignement classique au XIXe siècle.

2 Valdombre, "L'Appel de la race ou le roman canadien", Le Matin, 2 décembre 1922, 3. 
Mais le roman a particulièrement chatouillé celui que Lionel Groulx surnommera plus tard, dans ses Mémoires, le "prince des critiques d'alors ", l'abbé Camille Roy de Québec, qui publiait en décembre 1922, dans Le Canada français, "L'Appel de la raceRoman canadien", et en avril 1922 "Le Patriotisme de nos vieux maîtres" - $\bar{A}$ propos de L'Appel de la race ". Ces deux articles sont reproduits dans son ouvrage paru en 1924, $\dot{A}$ l'ombre des érables ${ }^{3}$, avec, en sus, le texte d'une conférence prononcée au Monument National de Montréal, le 6 avril 1915, conférence intitulée, comme par hasard, "Notre patriotisme littéraire en 1860 ".

Mon but n'est cependant pas de reprendre ici le récit de cette querelle. Je renvoie ceux que cela intéresse aux Mémoires de Lionel Groulx et à l'introduction que Bruno Lafleur a écrite pour l'édition du Nénuphar ${ }^{4}$. Je voudrais simplement dégager dans ce qui peut apparaître à plusieurs comme une banale querelle sur un roman à thèse, le niveau d'opposition qui la permit et la maintint aussi longtemps. La question qui se pose est la suivante : au nom de quels intérêts Camille Roy a-t-il réagi si violemment à la production d'un roman comme L'Appel de la race? Car, au-delà des considérations littéraires et para-littéraires - invraisemblance de l'intrigue, partialité de la peinture, incorrections de forme, mauvaise structure -, les interventions de Camille Roy reposent sur une problématique qui dépasse le roman, qui le précède et le suit à la fois. En effet, il faut voir la parution de L'Appel de la race comme l'occasion rêvée, pour le critique littéraire qu'était Camille Roy, de s'opposer aux thèses historiques soutenues par Lionel Groulx comme si elles étaient des défauts de composition ou des atteintes au vraisemblable. Ce que ne lui permettait pas la critique historique. Et de fait, l'opposition essentielle se situe au niveau des conceptions sur la questions nationale.

C'est ainsi qu'après les formules courtoises d'usage montrant que L'Appel de la race est "un ouvrage de haute valeur, et qui se détache en beau relief sur le fond trop plat de notre littérature romanesque $»^{5}$, Camille Roy attaque sur quatre fronts en même

3 Camille Roy, À l'ombre des érables, Hommes et livres (Québec, Imprimerie de l'Action sociale, Limitée, 1924), 348 [1] pages.

4 Bruno Lafleur, préface à l'édition de 1956, 9-93.

5 Camille Roy, op. cit., 273. 
temps. La première accusation concerne la théologie du père Fabien qui, sur la base du "volontaire indirect" encourage de Lantagnac à faire en Chambre un discours sur la question des écoles catholiques alors que sa femme, Maud Fletcher, a menacé de le quitter. L'utilisation du volontaire indirect qui renvoie à Maud Fletcher toute la responsabilité de son départ est, pour Camille Roy, une subtilité insoutenable. À Lionel Groulx, il oppose que

En épousant une Anglaise [de Lantagnac] a fixé à son patriotisme éventuel une limite qu'il ne peut plus moralement dépasser.

Il s'est mis dans l'impossibilité morale et pratique de rendre à la cause française les services qu'il voudrait maintenant lui rendre. Il y a comme cela des hommes qui ont brisé leur vie, et qui l'ont faite inapte à certains emplois, fussent les meilleurs. Tant pis! il ne leur est pas permis, pour essayer de réparer tant d'erreurs, de faire souffrir toute leur famille, et même par voie du "volontaire indirect" d'accabler des débris de leur foyer, femme et enfants. ${ }^{6}$

En fait, la théologie du père Fabien tend à privilégier ce que l'Église appelle la «société civile " sur la "société chrétienne » dont le mariage, indissoluble, inviolable et exempt de toute intervention politique, est un des piliers. Dissociant le politique du religieux, Camille Roy préfère sacrifier le premier au second. À son avis, la cause des écoles catholiques ne vaut pas un mariage chrétien, même avec une hérétique ou une étrangère. Telle est aussi la position officielle de l'Église catholique: le nationalisme doit contribuer au maintien de la société chrétienne et non l'inverse. Cela, Lionel Groulx ne le conteste pas, mais il refuse de subordonner le bien de la collectivité canadienne-française au succès d'un mariage mixte, d'autant plus qu'il considère ce type de mariage comme menaçant la survie de cette même collectivité. Et malgré l'intervention de l'abbé Villeneuve, jamais Camille Roy n'autorisera la rémission de sa faute à de Lantagnac.

La deuxième accusation découle de la première et la prolonge. En effet, si Camille Roy réagit aussi violemment au problème posé par la théologie du père Fabien, ce n'est pas seulement par respect pour le sacrement du mariage. C'est aussi parce qu'il croit ce

$6 \quad$ Ibid., 287. 
mariage viable malgré ce que Groulx appelle les atavismes de race. Il croit qu'avec tact et bonne volonté, Anglais et Français, à partir du moment où ils adoptent la même foi, peuvent constituer un ménage heureux: Jules de Lantagnac devait à Maud le respect de la race anglaise. De la même manière, alors que Groulx fonde le Canada sur la justice et l'équité de droit, Camille Roy propose l'amitié, la bonne volonté. L'un parle de contrat, l'autre de communion.

La troisième accusation renvoie au patriotisme de l'enseignement classique du $X^{\mathrm{e}}$ siècle. Fort de son expérience - n'a-t-il pas étudié à la même époque que de Lantagnac? - Camille Roy dit ne pouvoir accepter le jugement de Lionel Groulx lorsque celui-ci écrit que l'enseignement dans les collèges classiques n'a formé que des loyalistes, qu'il n'a pas su faire aimer le Canada pour lui-même et pour ses traditions françaises, qu'il n'a toujours célébré que les bienfaits de la constitution britannique et la loyauté des "pères " de la nation à la couronne d'Angleterre. L'accusation est double : elle touche non seulement le rôle de l'enseignement classique dans la tranmission des valeurs nationales, mais aussi le contenu de cet enseignement. À toutes ces mises en question, Camille Roy répond que l'enseignement de la loyauté envers le pouvoir établi est un bien moral incontestable et que, seule, une situation de grave injustice constitutionnelle - il insiste sur le mot - peut justifier la critique et la sécession. En effet, à Lionel Groulx qui prétend que la constitution britannique n'a pas eu que des bienfaits, Camille Roy répond que la Confédération garantit des libertés politiques et religieuses dont on pourrait apprécier la valeur si l'on comparait ce qui se passe ici à ce qui se passe en France où des frères de même race ne jouissent pas de ces libertés. D'autre part, s'il admet les lacunes de l'enseignement classique au XIXe siècle, Camille Roy rejette l'accusation de non-patriotisme: pour lui le militantisme se développe selon les besoins et on ne peut reprocher aux enseignants de cette époque de n'avoir pas su prévoir les problèmes constitutionnels des années 1920, comme la querelle des écoles catholiques et la reconnaissance des droits du français. C'est le roman et sa thèse mêmes qui sont remis en question: Camille Roy refuse de voir l'anglicisation de la bourgeoisie canadienne-française comme un signe de l'échec de la Confédération à permettre le développement d'une conscience nationale canadienne-française et comme la 
conséquence d'une soumission prêchée par le clergé au XIXe siècle. L'anglicisation de Jules de Lantagnac demeure pour lui une démission, voire une trahison personnelle, dont il est le seul responsable.

La quatrième accusation que fait Camille Roy à L'Appel de la race, celle d'employer l'expression "le Québec", peut sembler anodine. Mais l'histoire de la langue française au Québec et les derniers développements du discours nationaliste ont montré que derrière le neutralité apparente d'une expression comme "le Québec", qui s'oppose à "la province de Québec", se cache une perception, une volonté de parler d'un pays mieux défini, plus autonome, d'un pays différent qui détermine ses propres frontières. Le cas n'est pas nouveau: qu'on se souvienne du "Dominion du Canada" si farouchement défendu par les Orangistes. Camille Roy reproche donc à Lionel Groulx l'emploi de ce "barbarisme grammatical ", "fût-il autorisé par tous les journalistes du Devoir " ${ }^{7}$; il le condamne d'autant plus qu'il est parfaitement conscient de l'aspect volontaire et systématique de son utilisation et du danger de sa popularisation.

Je ne cherche pas à savoir qui de Camille Roy ou de Lionel Groulx a raison dans cette querelle. Cela dépend du point de vue. La querelle a ceci d'intéressant qu'elle oppose deux têtes de file des débats nationaux. Tous les deux sont nationalistes. Ce qu'ils ne partagent pas, c'est leur conception de la nation.

Pour Lionel Groulx comme pour Camille Roy, la nation se définit par des particularités fondées sur la psychologie, la langue, l'histoire, la religion et par un vouloir-vivre collectif. Le catholicisme est l'élément fondamental de la nation canadienne-française et la langue y est la gardienne de la foi. Mais, alors que Camille Roy distingue le politique du religieux, un peu à la manière d'Henri Bourassa, Lionel Groulx les associe. Chacun d'eux développera une thèse d'histoire qui aura comme but de légitimer leur position respective face à la nation dont ils parlent.

Ainsi, Lionel Groulx propose une vision quasi-mythique du Régime français où aurait existé une nation à l'état pur, déjà canadienne avant la Conquête. L'État canadien est pour lui un

7 Ibid., 295. 
arbitre qui s'engage à protéger les droits de chacune des deux nations constituant la patrie. C'est un contrat qui peut être rompu si les termes n'en sont pas respectés. Son flirt avec l'idéal de la Laurentie, État indépendant, avait comme effet de ressusciter le Québec comme entité politique et d'y concentrer tous les pouvoirs et les espoirs des francophones. S'il abandonne ce projet, Lionel Groulx n'en conserve pas moins un clivage complet entre Anglais et Français, comme en témoigne chacun des termes de la querelle.

Camille Roy développe, lui, une vision idéale du Régime anglais et du rôle providentiel de la Conquête face aux menaces de la Révolution française. Il partage la thèse des deux peuples fondateurs d'une nation à construire. L'État et la nation se confondent. Son opposition à l'emploi de l'expression "le Québec" vient de ce qu'il considère comme essentielle la dimension continentale du fait français en Amérique. De là son refus de centraliser ou de limiter au Québec l'action française, apostolat culturel et religieux; toute action politique, devant se faire, selon lui, dans le cadre de l'État-nation.

C'est pourquoi, sous le couvert de beaux principes, sous le couvert d'une banale querelle sur un roman à thèse, s'affrontent en réalité deux nationalismes : le nationalisme canadien-français et le nationalisme canadien. Aucun des éléments constitutifs de la querelle n'est vain, pas plus que les éléments du roman lui-même. La littérature ne véhicule pas des faits historiques vérifiables, mais, par la production de représentations imaginaires, elle propose des thèmes, des concepts, ce que d'autres appellent des visions du monde. Lionel Groulx le savait, lui qui donna au chapitre de ses mémoires sur la querelle de L'Appel de la race le sous-titre de "Propagande par le roman". Camille Roy le savait aussi, lui qui, dans ses critiques, ne s'attardait qu'aux éléments effectivement «subversifs» du roman de Lionel Groulx. 